\title{
Experimental Study on the Distribution Trends of Fouling on a Compressor Blade
}

\author{
Jie Tang $\mathbb{D},,^{1,2}$ Dianrong Gao $\mathbb{D},{ }^{1}$ Liwen Wang $\mathbb{D},{ }^{2}$ and Jinjian Huo $\mathbb{D}^{2}$ \\ ${ }^{1}$ Institute of Mechanical Engineering, Yanshan University, Qinhuangdao, China \\ ${ }^{2}$ Aviation Engineering Institute, Civil Aviation University of China, Tianjin, China \\ Correspondence should be addressed to Jie Tang; tangjie_cauc@163.com
}

Received 14 August 2020; Revised 19 October 2020; Accepted 16 November 2020; Published 28 November 2020

Academic Editor: Qiang Fu

Copyright (C) 2020 Jie Tang et al. This is an open access article distributed under the Creative Commons Attribution License, which permits unrestricted use, distribution, and reproduction in any medium, provided the original work is properly cited.

\begin{abstract}
The formation of a scale on a compressor blade surface is inevitable, and the study of the distribution of the scale can provide effective guidance for the cleaning of a wing engine. Using the waste liquid collected from the engine during the wing water washing process as a data sample, the main components of compressor blade surface fouling were analysed, which included $49.9 \% \mathrm{SiO}_{2}, 14.5 \% \mathrm{FeO}, 11.5 \% \mathrm{Al}_{2} \mathrm{O}_{3}, 9.4 \% \mathrm{CaO}$, etc. Based on JKR contact theory, a model for calculating the total thickness of the fouling layer on the blade surface was established. Through a simulation experiment on the fouling of a blade surface, the number of particulates deposited on the pressure surface was lower than the amount of the secondary deposition mass on the suction surface. From contrastive analysis of the results of perforation, the fouling is divided into three types: loose, dense, and transitional. The surface of a single blade can be divided into four different fouling areas. The parameters of the engine cleaning process can be designed according to the characteristics of the fourth area.
\end{abstract}

\section{Introduction}

An aero engine needs to continuously pull in air while functioning, and the suspended particulate pollutants in the air are inevitably be drawn into the engine and gradually adhere to the blade surface or edge to form fouling. The fouling on a blade surface has an important effect on the engine performance. Fouling results in surges in severe cases and poses hidden dangers to flight safety, which has aroused widespread concern.

Kurz et al. $[1,2]$ conducted an experimental study of fouling adhesion on the blade surface, quantitatively analysed the amount of fouling formed on blade surfaces under different conditions, and believed that the retention mode of particulate matter was related to the boundary layer shear stress. Osvaldo and Zuniga [3] found that oil residues caused by seals, steel, and aluminium particles generated by the wear of seal brushes and bearing parts and internal wear all contribute to fouling inside the compressor source. Tarabrin et al. [4] believed that due to the structural differences between the pressure surface and suction surface of the blades and the complex structure of the inner wall of the engine, the flow direction of the airflow changes, resulting in the accumulation of particulates trapped in the airflow. Song et al. [5] believed that the particles were mainly transported to the pressure surface of the blade under the action of inertial force, so the fouling on the pressure surface was mainly caused by larger particles. Yang and $\mathrm{Xu}$ [6] found in their research that the fouling of the leading edge of a blade is mainly affected by large particles, the mechanism of which is inertial impact, and the formation of a scale on the pressure area of the blade is mainly due to the impact of large particles after the deviation of the adjacent suction surface. Mathioudakis and Tsalavoutas [7] believed that the greater the relative humidity of the air is, the easier it is for water vapor to condense into a thick liquid film on the surface of the compressor blade, causing the dissolved particles to adhere to the blade 

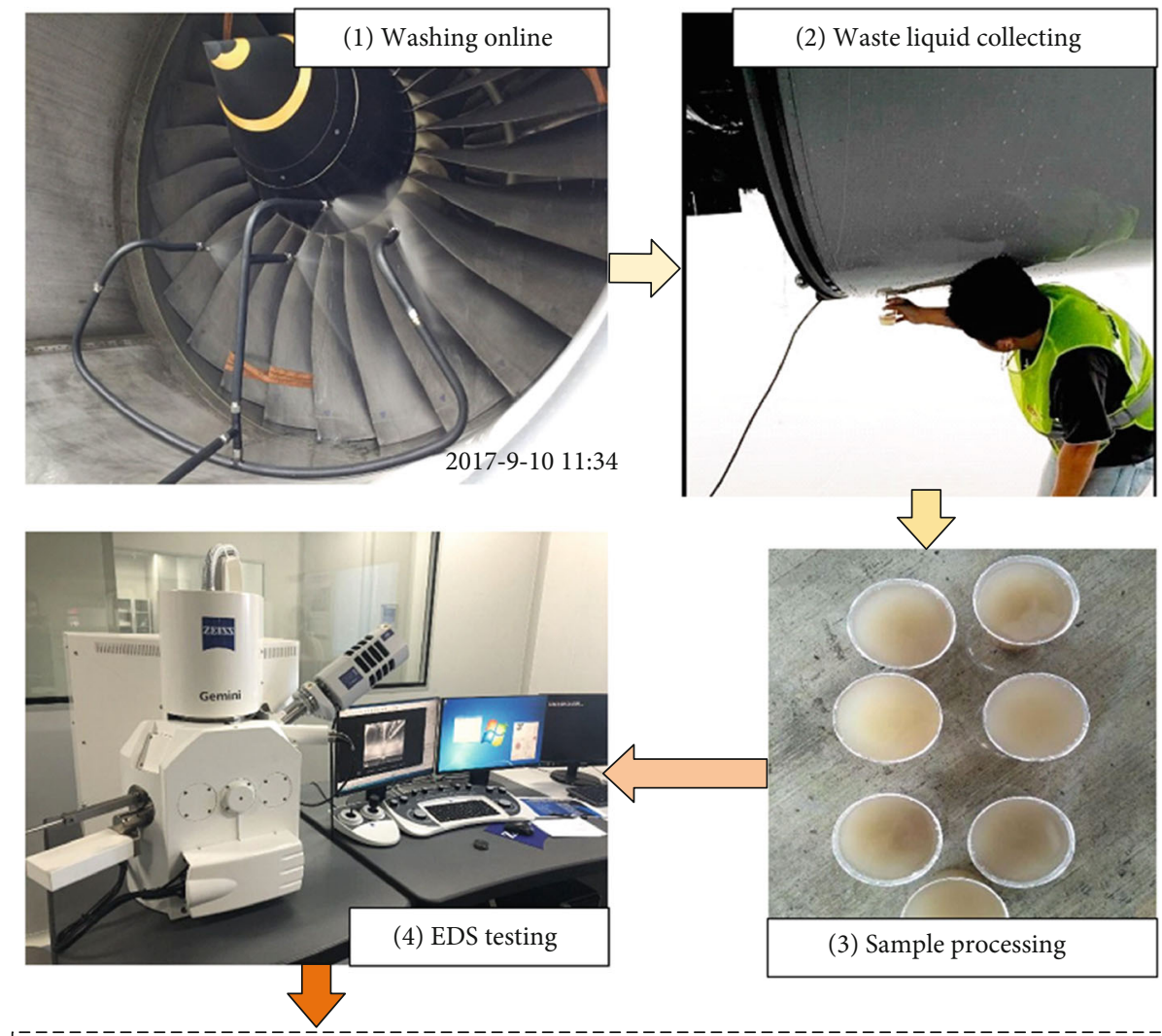

(3) Sample processing

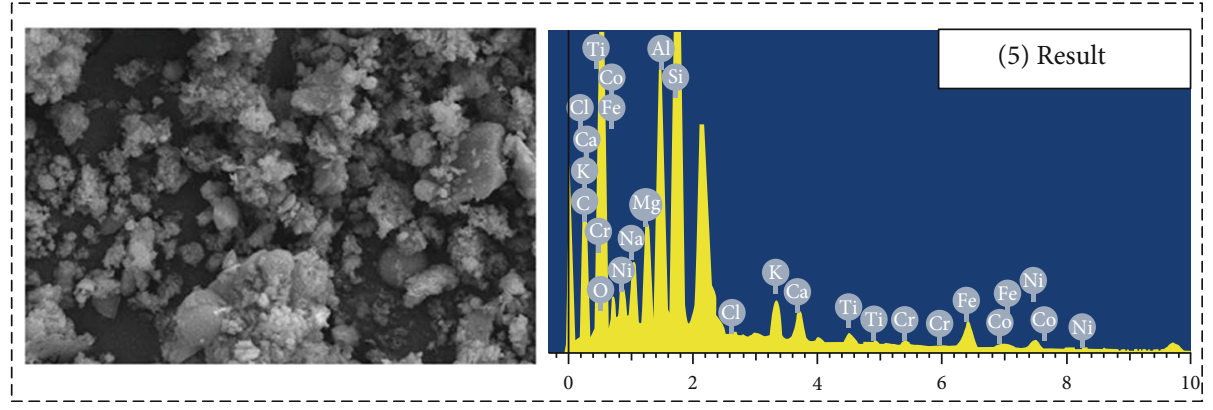

FIgURE 1: Collection and treatment of scale.

surface. Fielder [8] pointed out that natural environmental factors such as dense fog, rain, and snow and excessive humidity accelerate the adhesion process of particles. The size of the particles determines the movement trajectory of the particles and the influence of turbulent diffusion.

However, research on the distribution of scale on the blade surface still needs to be improved. First, the calculation model for the thickness of the scale deposited on the surface of a compressor blade has not been accurately judged. Additionally, the distribution of scale on the blade surface has not been quantified. Therefore, in this paper, research is carried out on the distribution trends of fouling mass and thickness calculation models for the surface of an engine compressor blade. The zoning method is used to analyse the changes in fouling mass and fouling characteristics, which provides guidance for the optimization of the parameters of the engine wing water washing process.
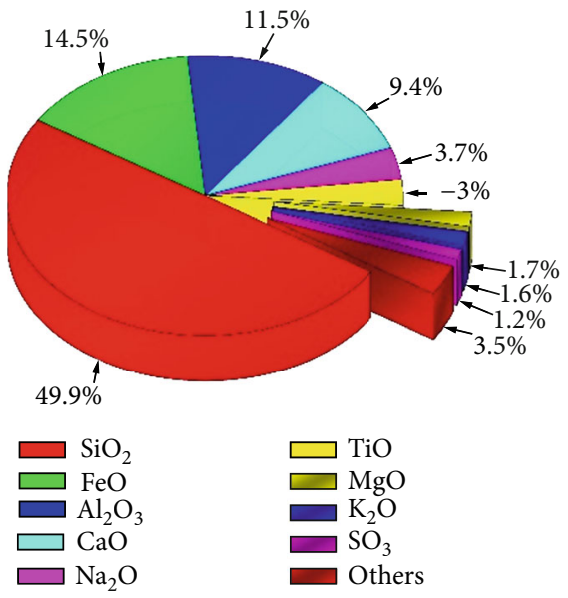

Figure 2: Compressor blade fouling. 


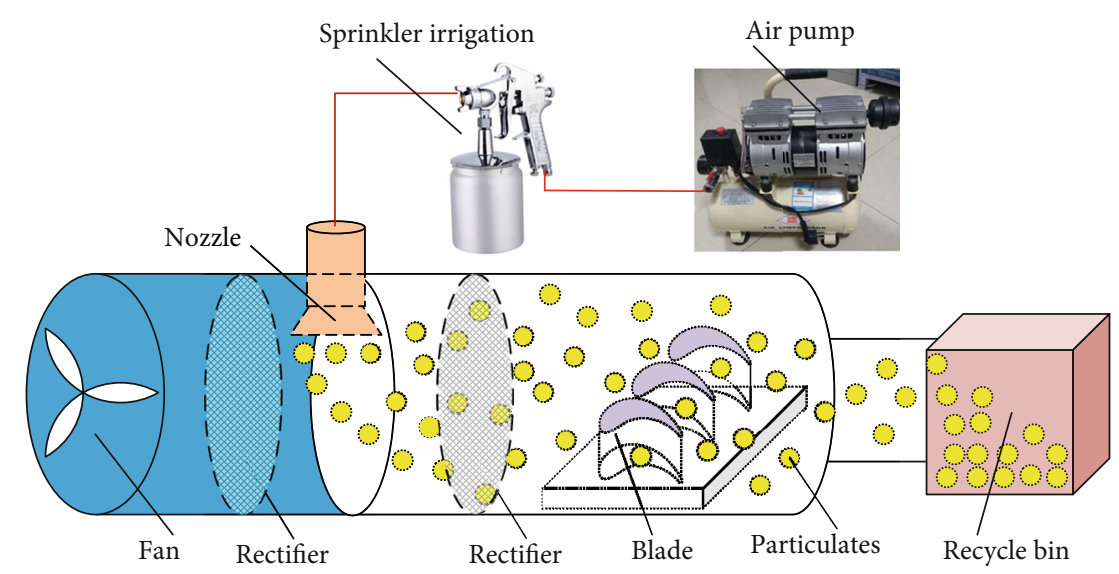

FIgURE 3: Schematic diagram of the experimental system.

\section{Collection and Analysis of Scale Samples on the Blade Surface}

The composition of the fouling on the blade surface has an important influence on the fouling rule. It is necessary to develop the sampling and analysis of the fouling on the blade surface to obtain the composition of the fouling. The cleaning of a wing engine provides a good opportunity to obtain scale samples from the surfaces of blades. A measuring cup is used to collect the cleaning waste liquid flowing out of the residual water hole of the engine. The sampling and analysis process of the waste liquid sample is shown in Figure 1. First, the sample is heated and dried to evaporate the water in the waste liquid, and finally, a scale sample of solid particles is obtained. Second, the glass sample is ground using a glass grinding rod and a grinding bowl to make the scale particles discrete, and finally, an energy dispersive spectrometer is used to measure and analyse the sample.

Through analysis of the scale samples, the scale components are obtained. The main elemental composition of scale includes $\mathrm{C}, \mathrm{N}, \mathrm{O}, \mathrm{Na}, \mathrm{Mg}, \mathrm{Al}, \mathrm{Si}$, and other components. According to the atomic mass fraction, the sample contains $\mathrm{CaO}, \mathrm{SiO}_{2}, \mathrm{MgO}, \mathrm{Al}_{2} \mathrm{O}_{3}$, etc., where $\mathrm{SiO}_{2}$ comprises $49.9 \%$, $\mathrm{FeO}$ comprises $14.5 \%, \mathrm{Al}_{2} \mathrm{O}_{3}$ comprises $11.5 \%, \mathrm{CaO}$ comprises $9.4 \%, \mathrm{Na}_{2} \mathrm{O}$ comprises $3.7 \%$, and $\mathrm{TiO}$ comprises $3 \%$. The remaining impurities account for $8 \%$, as shown by the detailed mass scores in Figure 2.

\section{Experiment on Fouling of Compressor Blade Surface}

3.1. Experimental System Construction. Figure 3 is a schematic diagram of a simulation experiment platform system for fouling on a compressor blade surface. The test system is composed of a fan, a rectifier net, an air pump, a pneumatic sprayer, a nozzle, a blade under testing, a particulate recovery box, and its culvert. The maximum air volume of the fan is $13980 \mathrm{~m}^{3} / \mathrm{h}$, and the speed is controlled by the inverter. During the experiment, the pneumatic sprayer sends the particulate matter into the experimental cavity through the nozzle.

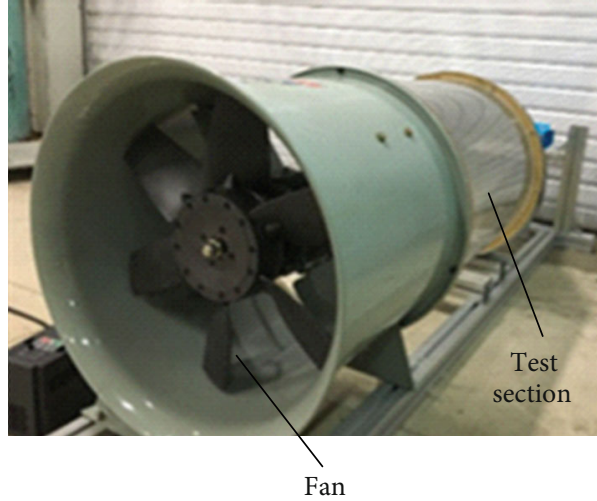

FIGURE 4: Experimental system physical map.

Under the action of the fan, the air velocity in the cavity is approximately $20 \mathrm{~m} / \mathrm{s}$. After passing through the rectifying mesh, the particulate matter enters the area of the tested blade and gradually forms a scale on the surface of the blade. After passing through the blades, the particulate matter enters the recovery box for collection. The undeposited particles are collected by the bag filter in the recovery box, and the airflow without particulate matter is discharged into the atmosphere.

The actual experimental system is shown in Figure 4.

3.2. Particulate Matter Ratio. The scaling of solid particles is completed in a gas-solid two-phase flow field. This system uses the blower process to simulate the gas-solid two-phase flow field. The experimental gas medium is air $\left(20^{\circ} \mathrm{C}\right)$ at normal temperature, its density is $1.293 \mathrm{~kg} / \mathrm{m}^{3}$, and its dynamic viscosity is $1.8 \times 10^{-5} \mathrm{~Pa} \cdot \mathrm{s}$.

According to the research results of the relevant literature [9], the size of the scale particles is $16 \mu \mathrm{m}$. The proportions of scale particles are $49.9 \% \mathrm{SiO}_{2}, 14.5 \% \mathrm{FeO}, 11.5 \% \mathrm{Al}_{2} \mathrm{O}_{3}$, and $9.4 \% \mathrm{CaO}$. The remaining scale components are replaced by water-insoluble $\mathrm{SiC}$ particles, so $14.7 \% \mathrm{SiC}$ particles are added. The composition ratio of particles in the scale simulation experiment is shown in Table 1. 
TABle 1: Composition ratio of particles in the experiment.

\begin{tabular}{lccccc}
\hline Composition & $\mathrm{SiO}_{2}$ & $\mathrm{FeO}$ & $\mathrm{Al}_{2} \mathrm{O}_{3}$ & $\mathrm{CaO}$ & $\mathrm{SiC}$ \\
\hline Ratio (\%) & 49.9 & 14.5 & 11.5 & 9.4 & 14.7 \\
\hline
\end{tabular}

\section{Theoretical Analysis of the Thickness of the Fouling Layer on the Blade Surface}

Solid particles are coupled with a variety of forces inside an engine and gradually deposit to form fouling. The deposition process can be divided into three stages: (1) The particles move with the air flow due to rotation. At this time, the particles are subject to drag forces and electrostatic forces, and thermophoretic forces, inertial centrifugal forces, and van der Waals forces change the speed of the particles. (2) The particles collide with the blade wall surface; some particles are gradually deposited to form a loosely deposited adhesion layer, and the others rebound from the surface, returning to the flow field at a certain speed. (3) During the accumulation stage of the scale layer, the loosely deposited adhesion layer continues to accumulate under the impact of temperature, pressure, and other solid particles, and phase change adhesion even occurs, forming a relatively dense dirt layer, as shown in Figure 5.

The movement of solid particles in the inner tract is a typical gas-solid two-phase flow. When the particles touch the surface of the blade, the particles themselves are deformed. The profile of the blade surface affects adhesion and binding. This belongs to the category of contact theory. Typical contact theories include Hertz contact theory, JKR theory, DMT theory, M-D theory, GW theory, and Persson theory [10].

The fouling process of the low-pressure compressor blade surface conforms to JKR contact theory. Combined with the research results of the literature ${ }^{11}$, the thickness $H$ of the scale deposit on the blade surface follows the following relationship:

$$
H=f\left(T, F, t, C, \sigma_{s}, \alpha\right) .
$$

In the formula, $T$ is the engine working time, $s ; F$ is the compressor air flow rate, $\mathrm{m}^{3} / \mathrm{s} ; t$ is the compressor working temperature, $\mathrm{K}$; $C$ is the particulate concentration, $\mathrm{kg} / \mathrm{m}^{3}$; $\sigma_{\text {s }}$ is the solid particle scaling stress, $\mathrm{Pa}$; and $\alpha$ is the contact angle between the solid particles and the blade surface and is dimensionless.

Let $d_{1}$ be the contact radius without considering the adhesion force, $d_{2}$ is the contact radius with the adhesion force considered, and the contact surface is equivalent to a circle with radius $d$, as shown in Figure 6. The solid particles in contact with the blade surface have a total energy of $U$ satisfying $U=f(A)$, where $A$ is the contact area and $A=\pi d^{2}$.

According to the contact area, the contact stress between the solid particles and the blade surface $\sigma_{j c}$ can be obtained:

$$
\sigma_{j c}=k \frac{P}{A} .
$$

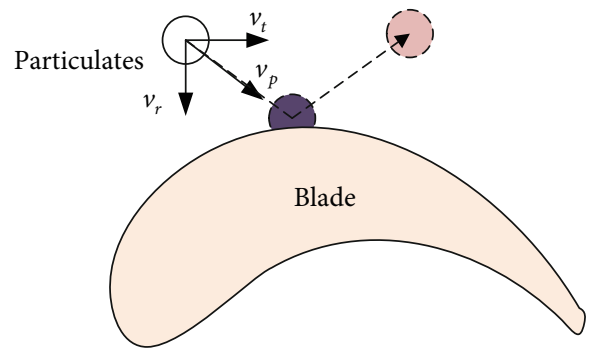

FIgURe 5: Particulate matter and blade surface contact process.

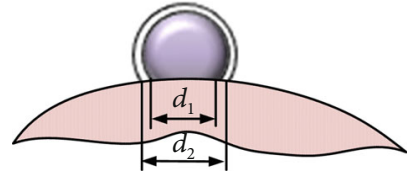

FIgURE 6: Schematic diagram of contact between particle and blade based on JKR contact theory.

TABLE 2: The mass of particles deposited on the blades.

\begin{tabular}{lcc}
\hline No. & $\begin{array}{c}\text { Pressure surface deposition } \\
\text { mass }(\mathrm{g})\end{array}$ & $\begin{array}{c}\text { Suction surface deposition } \\
\text { mass }(\mathrm{g})\end{array}$ \\
\hline 1 & 0.05304 & 0.06749 \\
2 & 0.05122 & 0.06607 \\
3 & 0.05200 & 0.06830 \\
4 & 0.05616 & 0.06983 \\
5 & 0.05685 & 0.07026 \\
\hline
\end{tabular}

In the formula, $P$ is the impact force between the particle and the blade, $\mathrm{N} ; k$ is the correction coefficient, $k=0.01 ; \sigma_{j c}$ is the contact stress between the particle and the blade, $\mathrm{Pa}$; and $A$ is the equivalent contact area between the particle and the blade surface, $\mathrm{m}^{2}$.

Under the condition of a certain temperature, the solid particles collide with the blade to produce contact stress. When the contact stress $\sigma_{j c}$ reaches or exceeds a certain critical value, the particles undergo plastic deformation, and the particles change from an ideal elastomer to an ideal plastic body. When the physical and mechanical properties of the particles are consistent with an ideal plastic body, the critical value is defined as the scaling stress $\sigma_{s}$ of the particles. After the particulate matter is combined with the blade to form fouling, the contact area is $A_{1}$, and then the single solid particulate and the blade surface form a fouling thickness of height $h$.

$$
h=\frac{V}{A_{1}} .
$$

The total thickness of the scale layer on the blade surface can be expressed as

$$
H=\sum_{i=1}^{N} h_{i}
$$


In the formula, $A_{1}$ is the contact area of single particles to form the scale, $\mathrm{m}^{2} ; V$ is the volume of solid particles, $\mathrm{m}^{3} ; h$ is the thickness of the sscale layer formed by single particles, $m$; $h_{i}$ is the thickness of the scale layer formed by different particles, $\mathrm{m} ; N$ is the number of particles deposited in the same area of the blade; and $H$ is the total height of the scale layer formed by $N$ particles deposited in the same area on the surface of the blade, $\mathrm{m}$.

\section{Analysis of Results}

There are a total of three blades in the experimental system. Considering the influence of the boundary layer in the simulation process of scaling, only the intermediate blades are selected for the analysis of the experimental results to ensure the rationality of the experimental results. In this experiment, a total of 5 full-scale simulation experiments are carried out. After each experiment, the sediment on the surface of the middle blade is measured and analysed, and the blade is cleaned again and then dried for the next experiment.

5.1. Deposition Mass Measurements. The deposition mass is an important indicator for measuring particle deposition. The deposition mass of the scale on the surface of a single blade is very small, generally on the order of $10^{-2} \mathrm{~g}$. In the experiment, a 0.00001 electronic balance is used to measure the deposition mass to ensure the accuracy of the experiment.

In the experiment, the air volume of the fan is set to $800 \mathrm{~m}^{3} / \mathrm{h}$, the particle concentration is adjusted to $50 \mathrm{~g} / \mathrm{m}^{3}$ through a powder feed sprayer, and the results of the five experiments are weighed. The deposition amount on the blade surface is shown in Table 2. The standard deviation of the mass of the deposition on the surface of the blades is small, below $10^{-3}$.

It can be seen from the measurement results that the number of particulates deposited on the pressure surface is lower than the secondary deposition mass of the suction surface; the average value of the particulate deposition on the pressure surface is $0.05385 \mathrm{~g}$, the average value of the deposition mass of the suction surface is $0.06839 \mathrm{~g}$, and the difference between the scale accumulation on each side of the blade is $0.01454 \mathrm{~g}$. Figure 7 shows a comparison of the amounts of scale deposits on the blade surface.

5.2. Observation of Sediment Distribution Patterns. The distribution of solid particles on the compressor blades is an important part of the study of particle deposition. Using a camera to photograph the scaled blades, the distribution of sediment particles on the blade surface, as shown in Figure 8, is obtained. Comparing the images of the five experiments, it can be found that the particle deposition morphology on the blade surface is very similar, so the difference between the blades can be ignored, and a morphological analysis is performed based on the results of a single experiment.

5.3. Contrastive Analysis of Penetration Testing. Penetration is an important means of nondestructive testing of the engine state in the wing. A flexible probe with an optical lens is sent into a borehole, and an internal image of the engine is obtained using optical imaging principles, which is then ana-

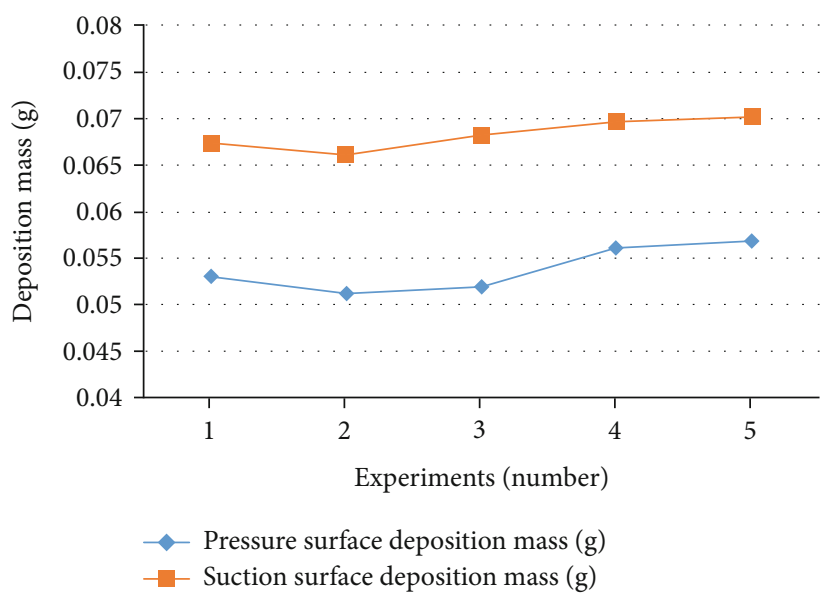

Figure 7: Comparison of the scale qualities of the blade surface areas.

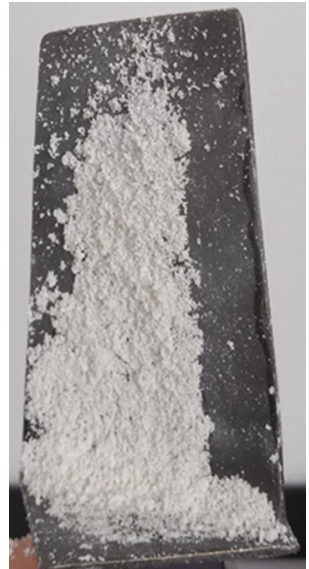

Pressure surface

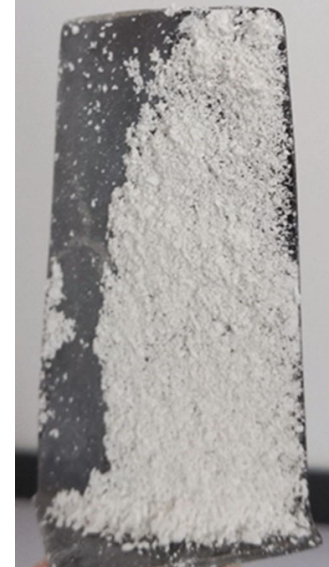

Suction surface
FIGURE 8: Observation of the surface morphology of the compressor blade surface area.

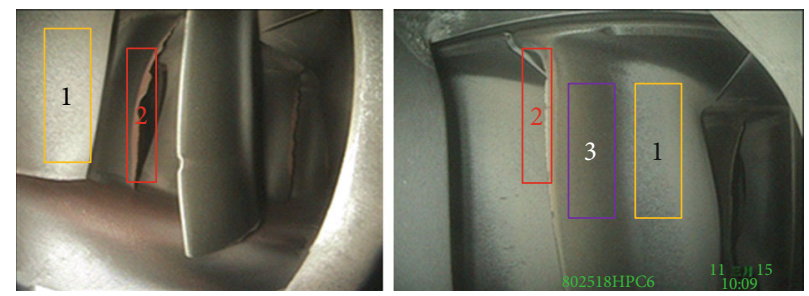

FIGURE 9: Dirt classifications.

lysed and evaluated regarding the status of the engine. This provides a decision-making basis for fault diagnosis and maintenance plan formulation.

Figure 9 shows the results of compressor blade borehole inspection. It can be seen from the figure that the fouling is mainly concentrated on the trailing edge of the blade and the root area of the blade. A large amount of accumulated fouling occurs on the trailing edge of the blade, and the fouling layer of the blade is relatively thin. Analysis shows that 


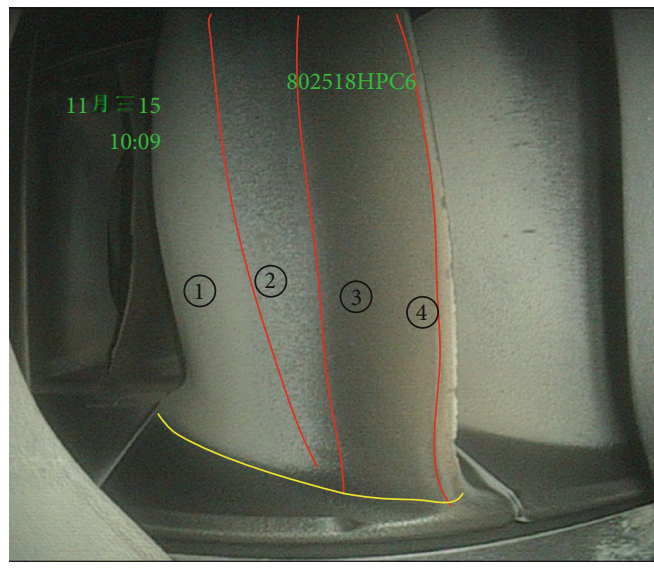

Figure 10: Pressure surface dirt zones.

the scale is divided into three types: loose type, dense type, and transitional type. As shown in Figure 9, No. 1 is the loose type, No. 2 is the dense type, and No. 3 is the transitional type.

The scaling of a single blade can be divided into four different fouling areas through analysis, as shown in Figure 10. Area 1 is the leading edge fouling layer, and the main feature of this area is that the fouling layer is thin and dense. Area 2 is the transitional scale layer in the middle of the blade, which is characterized by a sparse and discontinuous scale. Area 3 is a trailing edge scale layer, which is characterized by a firm but sparse scale. The trailing edge scale area is the 4 th area, and the scale layer is thick, with massive amounts of accumulation and high hardness.

To achieve complete cleaning, the energy of the cleaning fluid must exceed the dirt binding energy. According to the dirt distribution characteristics on the surface of the blade, the dirt binding energy in the 4th zone is the highest. This area is cleaned, so in the design process of the scale cleaning process parameters, the design calculations are mainly carried out for the scale 4 area to ensure the complete removal of all the scales on the surface of the compressor blade.

\section{Conclusion}

In this paper, in accordance with the design requirements of the on-wing engine cleaning process parameters, the trends of surface area fouling of compressor blades were studied:

(1) Using a surface sample from the compressor blade surface, the composition of the fouling on the aero engine compressor blade surface was obtained, which included $\mathrm{SiO}_{2} 49.9 \%$, FeO 14.5\%, $\mathrm{Al}_{2} \mathrm{O}_{3} 11.5 \%, \mathrm{CaO}$ 9.4\%, $\mathrm{Na}_{2} \mathrm{O} 3.7 \%$, $\mathrm{TiO} 3 \%$, and remaining impurities $8 \%$

(2) A theoretical analysis was carried out for the thickness of the fouling layer on the surface of a compressor blade. Based on JKR contact theory, the factors affecting the fouling thickness on a blade surface were analysed
(3) Through a simulation experiment of particulate fouling on the surface of compressor blades, it was found that the number of particulates deposited on the pressure surface was lower than the secondary deposition mass of the suction surface, and the difference between the fouling deposition on each side of the blade was $0.01454 \mathrm{~g}$. Compared with the results of engine drilling, the dirt was divided into three types: loose type, dense type, and transitional type. The surface of a single blade can be divided into four different fouling areas. The parameters of the engine cleaning process can be based on the characteristics of the fourth area

\section{Data Availability}

The data used to support the findings of this study are available from the corresponding author upon request.

\section{Conflicts of Interest}

The authors declare that they have no conflicts of interest.

\section{Acknowledgments}

The authors are thankful for the teacher's guidance and modifications to make this paper more perfect. This thesis was supported by the National Natural Science Foundation of China (U1733201, 51705518).

\section{References}

[1] R. Kurz, G. Musgrove, and K. Brun, "Experimental evaluation of compressor blade fouling," in Volume 9: Oil and Gas Applications; Supercritical CO2 Power Cycles; Wind Energy, Seoul, South Korea, 2016.

[2] R. Kurz and K. Brun, "Fouling mechanisms in axial compressors," Journal of Engineering for Gas Turbines and Power, vol. 134, no. 3, article 032401, 2012.

[3] M. Osvaldo and V. Zuniga, Intake Systems for Industrial Gas Turbines, Cranfield University, 2002, 2003.

[4] A. P. Tarabrin, V. A. Schurovsky, A. I. Bodrov, and J. P. Stalder, "An analysis of axial compressor fouling and a blade cleaning method," Journal of Turbomachinery, vol. 120, no. 2, pp. 256-261, 1998.

[5] T. W. Song, J. L. Sohn, T. S. Kim, J. H. Kim, and S. T. Ro, “An analytical approach to predicting particle deposit by fouling in the axial compressor of the industrial gas turbine," Proceedings of the Institution of Mechanical Engineers, Part A: Journal of Power and Energy, vol. 219, no. 3, pp. 203-212, 2005.

[6] H. Yang and $\mathrm{H}$. Xu, "The sensitive parameter study of axial flow compressor fouling," Research Journal of Applied Sciences Engineering and Technology, vol. 5, no. 10, pp. 3057-3062, 2013.

[7] K. Mathioudakis and A. Tsalavoutas, "Uncertainty reduction in gas turbine performance diagnostics by accounting for humidity effects," Journal of Engineering for Gas Turbines and Power, vol. 124, no. 4, pp. 801-808, 2002.

[8] J. Fielder, "Evaluation of zero compressor wash routine in RN service," in ASME Turbo Expo 2003, collocated with the 2003 
International Joint Power Generation Conference, pp. 543-547, Atlanta, Georgia, USA, 2003.

[9] Y. Xiaojun and Z. Jiaxiong, "Numerical simulation of particle deposition process inside turbine cascade," Acta Aeronautica et Astronautica Sinica, vol. 38, no. 5, pp. 120-130, 2017.

[10] S. F. Chen, J. J. Wang, and Y. H. Jin, "Model of particle deposition and adhesion on blade surface of flue gas turbine (in Chinese)," The Chinese Journal of Process Engineering, vol. 18, no. 3, pp. 447-453, 2018. 